\title{
Metapher als Entzug - Metapher als Gabe: Wittgenstein mit Blumenberg lesen?
}

\author{
Matthias Kroß, Berlin
}

Obgleich sich Ludwig Wittgenstein Zeit seines Lebens mit dem philosophischen Problem der Bedeutung sprachlicher Zeichen beschäftigt hat, sind in seinen Schriften doch nur wenige Bemerkungen zur Metapher zu finden. Dies verblüfft umso mehr, als Wittgensteins Aufzeichnungen und Bücher dem Leser ein wahres Füllhorn an metaphorischen Wendungen und allerlei anderen Sprachbildern bieten. Im Folgenden soll freilich nicht der Versuch unternommen werden, Gründe für das Fehlen einer Reflexion über Metaphern bei Wittgenstein zu suchen, sondern der Vorschlag unterbreitet werden, durch eine philosophisch-metaphorisierende Lesweise Wittgensteins einen anderen Zugang zu seinem Werk zu suchen. Dafür scheinen die Arbeiten Hans Blumenbergs zur philosophischen Bedeutung der Metapher in hohem Maße geeignet zu sein, auch wenn sie am Ende noch zu sehr dem Husserl'schen Lebensweltkonzept verpflichtet bleiben, um ohne Modifikation auf Wittgensteins Denkbewegungen angewandt werden zu können. In einer modifizierten Fassung hingegen lässt sich meines Erachtens mit Blumenbergs Ansatz eine z.T. neue und fruchtbare Deutung der Wittgensein'schen Philosophie skizzieren.

I

Als Hans Blumenberg (1920-1996) zur konzeptionellen Mitarbeit am Historischen Wörterbuch der Philosophie eingeladen wurde, schlug er vor, nicht nur das Lemma Metapher aufzunehmen, sondern umgekehrt etliche Metaphern zu Lemmata zu machen. Bekanntlich wurde sein Ansinnen vom Herausgeberkreis abgelehnt, mit höflichem Bedauern, doch mit einiger Entschiedenheit:

Der Herausgeberkreis hat, nicht leichten Herzens, darauf verzichtet, Metaphern und metaphorische Wendungen in die Nomenklatur des Wörterbuches 
aufzunehmen, obwohl ihm klar war, dass, wie H. BLUMENBERG gezeigt hat, gerade die der Auflösung in Begrifflichkeit widerstehenden Metaphern „Geschichte“ in einem radikaleren Sinn als „Begriffe“ haben und an die „Substruktur des Denkens heranführen, die die „Nährlösung der systematischen Kristallisationen“ ist. Ritter 1971, VIIf.)

Mittlerweile ist das Wörterbuch abgeschlossen. Die Vorahnung seiner Herausgeber, dass sich das Ausklammern des Metaphorologischen aus dem Wörterbuch als eine offene Flanke des Gesamtprojekts erweisen würde, hat sich durchaus bewahrheitet: der von ihnen ausgeschlossene Aspekt einer metaphorologisch inspirierten begriffskritischen Betrachtung der Philosophie hat, wenn auch mit einiger zeitlichen Verzögerung, stark an Bedeutung gewonnen. 2004 hat Bernhard W. F. Taureck den ersten Band eines auf drei Bände angelegten hermeneutischen Großprojekts zur Rolle von Metaphern der Philosophie vorgelegt (Taureck 2004), und seit 2007 bietet das Wörterbuch der philosophischen Metaphern einen zumindest exemplarischen Überblick über die Leitmetaphern der Disziplin (Konersmann 2007). Mit der starken Rezeption der Postmoderne und des Dekonstruktivismus hat die Metaphernforschung zweifellos einen weiteren Schub erhalten. ${ }^{1}$

Im englisch-amerikanischen Raum scheint das Interesse an der Metapher vor allem über die Literaturtheorie zum Gegenstand des philosophischen Interesses geworden zu sein; in die analytische Philosophie hat die Diskussion vor allem seit dem 1976 erstmals publizierten MetaphernAufsatz von Donald Davidson Eingang gefunden (Davidson 1986). Davidsons Aufsatz war eine Reaktion auf die vielen vergeblichen Bemühungen vor allem innerhalb der angelsächsischen Philosophie, entweder die Logik der Metaphern unter Verwendung des Begriffs der Bedeutung aufzuklären oder eine Theorie der Metapher zu entwickeln. Das „Wunder“ der Bedeutungsübertragung, welches die Metapher nach landläufiger Vorstellung vollbringe, lässt sich laut Davidson per definitionem nicht aufklären -

${ }^{1}$ Neben den Arbeiten Blumenbergs wurde die Diskussion um die Rolle der Metaphern in der Philosophie im deutschsprachigen Raum durch die hermeneutisch orientierten Arbeiten Paul Ricœurs (dt. 1986) oder Ernesto Grassis (Grassi 1992) weitergeführt. Die Rezeption des Dekonstruktivismus im deutschsprachigen Raum wurde wesentlich von Anselm Haverkamp befördert (vgl. Haverkamp 1998 und Haverkamp 2007). 
schließlich unterstelle man mit der Definition der Metapher als übertragene Bedeutung einer literalen Ursprungsbedeutung bereits jenes Paradox, das man hinterher verzweifelt und erfolglos aufzulösen versuche. Gordische Knoten lassen sich bekanntlich nicht lösen, sondern allenfalls zerschlagen. (Es wäre gewiss hierbei reizvoll zu untersuchen, was Wittgenstein meinen könnte, wenn er von den „Verknotungen des Verstandes“" spricht).

Jens Kertscher hat zu Recht angemerkt, dass Davidsons konstative Gebrauchstheorie der Metapher um ein pragmatisch-praktisches Element bereichert werden muss, um im Ergebnis mehr zu sein als nur eine Trivialität (wobei der Akt der Trivialisierung durchaus nicht-trivial ist: darin besteht Davidsons Leistung). Diese Ergänzung um die gesellschaftliche Praxis, die Gepflogenheiten einer Sprachgemeinschaft, sei notwendig, so Kertscher, wenn man etwa zwischen Gelingen und Misslingen eines metaphorischen Gebrauchs in der Sprache unterscheiden möchte. Zudem können Metaphern absterben und zu Vollbürgern der Terminologie werden (vgl. Kertscher 2004).

Davidsons sprachanalytischer Entlüftung diverser philosophischer Theorieblasen kann ich durchaus folgen. Dennoch hinterlässt seine Untersuchung vielleicht nicht nur bei mir einen schalen Nachgeschmack. So wirken seine Beispiele und die Erläuterungen zu ihnen zuweilen farblos oder geradezu nichtssagend, etwa wenn er T.S. Eliots Gedicht The Hippopotamos auf ein Ensemble teils propositional wahrer, teils faktisch falscher Aussagen reduziert - soviel Alltagsverstand ist für den Leser nur erträglich, wenn man die gute philosophische Absicht des Interpreten in Anschlag bringt: eben gegen bestimmte unangemessene Theorien $\mathrm{zu}$ argumentieren. Er selbst begnügt sich damit, den sprachlichen Gehalt von Eliots Gedicht so zu charakterisieren: „Freilich wird in dem Gedicht vieles angedeutet, was über die buchstäbliche Bedeutung der Wörter hinausgeht. Doch Andeutung ist dasselbe wie Bedeutung.“ (Davidson 1998, 65) Mit anderen Worten: Wohl hat die Metapher stets etwas Betörendes, weil rhetorisch Überschießendes (also An-Deutendes), aber da An-Deuten bereits Bedeuten ist, gäbe es hier erst gar kein wirkliches Problem. Das vielen Philosophen so teure Aufspüren verborgener Bedeutung ist damit philosophisch miterledigt.

Zur Verblüffung des Lesers hindert dies Davidson allerdings nicht, in einer ebenso knappen wie beeindruckenden Schlusspirouette am Ende 
seiner Ausführungen feierlich zu verkünden, dass es so etwas wie „die verborgene Kraft der Metapher“ (ebd., 75) gäbe. Damit dementiert er am Ende das Ziel, zu dem er zu Beginn seiner Ausführungen aufgebrochen war. Und der Leser fragt sich, ob Davidson mit dieser „Kraft“ nicht gleichsam eine virtus metaphorica postuliert - in Analogie zu Nietzsches sarkastischem, aber durchaus zutreffendem Diktum, dass Kants Ableitung der Verstandesvermögen ,vermöge eines Vermögens“ an die Herleitung der Wirkung eines Schlafmittels vermöge einer virtus dormitiva erinnere (Nietzsche 1978, I, 11).

\section{II}

Da Davidsons virtus metaphorica als Schlussstein seines Argumentationsbogens kaum zu überzeugen vermag, könnte man seinen Aufsatz trotz der Vielzahl von Kommentaren, die er in der englischsprachigen Philosophie ausgelöst hat, getrost zur Seite legen, wenn sein erratischer Schluss nicht zugleich etwas für die Metaphorologie Interessantes anzeigen würde. Es ist klar, dass Davidson das Geheimnis der Metapher natürlich keineswegs dadurch löst, dass er es in die Metapher zurückbiegt, indem er nachweist, dass aus ihr keine spezifische Bedeutungstheorie herauszuholen ist. Die von ihm vollzogene Inklusion des Metaphorischen in Sprache und deren propositionale Grundstruktur (jedenfalls bestimmt Davidson die Metapher ausschließlich in Relation zu dieser Struktur) wirkt deshalb befremdend, weil laut Davidsons eigenem Bekunden durch die Metapher doch etwas zur Sprache kommen soll, was sprachlich gerade nicht zu erwarten ist. Die Metapher erbringt eine Leistung, die sich in Sprache zeigt, deren Ursprung aber noch nicht auf der Oberfläche der propositionalen Sprache zu verorten ist. Davidsons Einschluss der Bedeutung des Metaphorisierens in die Sprache impliziert also in einem Zug die konträre Geste des Ausschlusses eines wichtigen Aspekts des metaphorischen Redens aus der Sprache. - Aber was wird hier durch Einschluss gerade ausgeschlossen?

Davidson selbst untersucht in seinem Text lediglich die (von ihm zu Recht als unzureichend erachteten) Antworten seiner angelsächsischen Kollegen. Andere interessante Antworten hat aber die von der analytischen Philosophie oftmals so fahrlässig ignorierte Tradition des historischhermeneutischen Denkens zu geben versucht (zu den Hintergründen dieser 
Vernachlässigung vgl. neuerdings Glock 2008). An erster Stelle ist der bereits eingangs zitierte Hans Blumenberg zu erwähnen - leider ein in analytischen Kreisen immer noch weitgehend Unbekannter, obwohl er doch Zeit seines Lebens sich dem Thema der Metapher gewidmet hat und eine stolze Sammlung monumentaler Studien zu einzelnen Leitmetaphern der abendländischen Philosophiegeschichte vorgelegt hat. Blumenberg sticht auch deshalb wohltuend in dem Strauß möglicher nicht-davidsonistischer Antworten hervor, weil er, im Unterschied zu metaphysisch euphorisierten Autoren wie Paul Ricœur (Ricœur 1986), Ernesto Grassi (Grassi 1992) oder George Steiner (Steiner 1990) - sich selbst als Historiker und Philologe verstand (eine Qualifikation, die man bei Davidson und anderen analytischen Philosophen zuweilen schmerzlich vermisst).

Nachdem er, wie oben erwähnt, im Wörterbuch keine Aufnahme gefunden hatte, hat Blumenberg gleichsam auf eigene Faust verschiedene Grundmetaphern des abendländischen Denkens geduldig in ihrer historischen Vielfalt untersucht und in dickleibigen Büchern nachvollzogen. Was er aber im Grunde seines Herzens zu zeigen versuchte, ist ein „Ausblick auf eine Theorie der Unbegrifflichkeit", die er in seinem Buch Schiffbruch mit Zuschauer. Paradigma einer Daseinsmetapher umrissen hat (Blumenberg 1979). Diese verweist wiederum auf eine erstmals in Rothackers $A r$ chiv für Begriffsgeschichte 1960 erschienene Studie zu Paradigmen einer Metaphorologie (Blumenberg 1960/1999) zurück, auch wenn Blumenberg 1979 seine Frühschrift in mancher Hinsicht verändern sollte. In seinem grundlegenden Aufsatz von 1960 hatte Blumenberg im Anschluss an Nietzsche die Metaphern als gleichsam plasmatische Vorformen von Begriffen in den Tiefen des Denkens bezeichnet, die an der Oberfläche der Lebenswelt zu fixen Termen auskristallisieren und als Metaphern am Ende absterben, weil sie bedeutungsgehärtet in das Begriffsvokabular der Sprache eingingen. Mit diesem Gedanken knüpft er direkt an Nietzsche an. Das „bewegliche Heer von Metaphern“, das die Unstetigkeit individueller Erfahrungstatbestände spiegelt, so Nietzsche, petrifiziert evolutionär zu einem großen und starren Bau der Begriffe:

Während jede Anschauungsmetapher individuell und ohne ihresgleichen ist und deshalb allem Rubrizieren immer zu entfliehen weiß, zeigt der große Bau der Begriffe die starre Regelmäßigkeit eines römischen Kolumbariums und 
atmet in der Logik jene Strenge und Kühle aus, die der Mathematik zu eigen ist. (Nietzsche 1984, 880)

Blumenberg bemerkt allerdings schon 1960, dass es eine bestimmte Gruppe von Metaphern gibt, die sich nicht zu Begriffen verfestigen, weil sie ihrerseits gleichsam die Bedingung der Möglichkeit für die Festlegung von Begriffsbedeutungen sind. Er nennt sie ,absolute Metaphern“. Sie sind weder angemessen noch unangemessen - sie sind vielmehr die Voraussetzung dafür, dass wir über den falschen oder richtigen Gebrauch von Begriffen entscheiden können. Absolute Metaphern stellen das Instrumentarium der philosophischen Operationen dar - als Beispiele nennt Blumenberg Begriffe wie ,Welt', ,Geschichte', ,Bewusstsein', oder solche Begriffe wie ,Begriff‘, ,Urteil‘, ,Logos‘ etc.

Dass die Metaphorizität dieser Metaphern in der Philosophie und Theorie so häufig übersehen, ja in der Metaphysik geradezu verleugnet wird, ${ }^{2}$ leitet Blumenberg, hier wiederum in enger Anlehnung an Nietzsche, von einer evolutionären Anthropologie der prekären Selbstbehauptung her. Nietzsche hatte in Über Wahrheit und Lüge als Grund für das „Übersehen“ der begriffskonstitutiven Leistung der Metapher erkannt, dass der Mensch

[n] ur durch das Vergessen jener primitiven Metapherwelt, nur durch das Hartund Starrwerden einer ursprünglichen, in hitziger Flüssigkeit aus dem Urvermögen menschlicher Phantasie hervorströmenden Bildermasse, nur durch den unbesiegbaren Glauben, diese Sonne, dieses Fenster, dieser Tisch sei eine Wahrheit an sich, kurz nur dadurch, daß der Mensch sich als Subjekt, und zwar als künstlerisch schaffendes Subjekt, vergißt, er mit einiger Ruhe, Sicherheit und Konsequenz [lebt]: wenn er einen Augenblick nur aus den Gefängniswänden dieses Glaubens herauskönnte, so wäre es sofort mit seinem „Selbstbewusstsein“ vorbei. (Nietzsche 1984, 880)

In Blumenbergs Adaption liest sich dieser Gedanke folgendermaßen:

Das animal symbolicum beherrscht die ihm genuin tödliche Wirklichkeit, indem es sie vertreten läßt; es sieht weg von dem, was ihm unheimlich ist, auf das, was ihm vertraut ist. Am deutlichsten wird das dort, wo das Urteil mit seinem Identitätsanspruch überhaupt nicht ans Ziel kommen kann, entweder

${ }^{2}$ Diesen Vorgang konstatieren auch Heidegger und Derrida, die den „normalphilosophischen“"Metapherngebrauch als ausschließlich der Metaphysik zugehörig begreifen. 
weil sein Gegenstand das Verfahren überfordert (die „Welt“, das „Leben“, die „Geschichte“, das „Bewusstsein“) oder weil der Spielraum für das Verfahren nicht ausreicht, wie in Situationen des Handlungszwanges, in denen rasche Orientierung und drastische Plausibilität vonnöten sind. (Blumenberg 1981, 116)

$\mathrm{Ob}$ sich mit dieser evolutionär-anthropologischen Transposition des nietzscheanischen Gedankens eine überzeugende Theorie des Unbegrifflichen begründen lässt, muss allerdings bezweifelt werden. Blumenberg diagnostiziert einen Bedarf an Metaphern für drei Lebenssituationen: (1) die Identitätslogik ,normaler“ Urteile versagt angesichts einer feindlichen und lebensbedrohlichen Umwelt (,genuin tödliche Wirklichkeit"'); (2) die Empfindung der ,Unheimlichkeit“" etwa der „Welt“ oder des „Bewusstseins“, führt zu einem symbolischen Vertretungshandeln, das Unheimlichkeit in Vertrautheit zurückverwandelt; (3) Reaktionen auf Umweltzwänge, die keine Spielräume für reflexiv gesicherte Urteile zulassen, sondern ,rasche Orientierung“" und ,drastische Plausibilität“" verlangen.

Es ist leicht zu sehen, dass sich Blumenberg mit seinen Argumenten in einen anthropologischen oder, zeitgeistgemäßer ausgedrückt, in einem evolutionsbiologischen Zirkel zu verstricken droht. Dieser besteht darin, dass aus dem evolvierten Sachverhalt auf seine evolutionäre Zweckmäßigkeit geschlossen, dass seiner Funktion eine heimliche Teleologie beigelegt wird. So wie es einem Beobachter ex post zweckmäßig erscheinen mag, dass die Menschen irgendwann begannen, aufrecht zu gehen, um die Hände zur Umgestaltung ihrer Umwelt frei zu haben, so wird einem Blumenbergschen Beobachter das Symbol als tauglich erscheinen, um eine prekäre oder sogar ,genuin tödliche“" Wirklichkeit zu bemeistern. - Sollte man daraus schließen, dass die Menschen am Ende an Symbolversagen sterben? Ein ähnlicher und nicht minder störanfälliger Suggestivschluss liegt bereits Husserls Konzeption der „Widerstimmigkeit“ der Intentionalität zugrunde, auf sich die Blumenbergs beziehen wird. Für Husserl bedeutet intentionale Einstimmigkeit das Eingelassensein des Bewusstseins in die Lebenswelt, mit der Folge, dass das (unvermeidliche) Ereignis der „Störung“ dieser Stimmung, die ,Widerstimmigkeit“", zu dem Impuls führt, zur Ein- und Normalstimmigkeit höherer Ordnung zu gelangen. Der damit ausgesprochene Vorrang der Lebenswelt gegenüber den die Lebenswelt transzendierenden begrifflichen Bestimmungen in Wissenschaft und Theorie führt Husserl jedoch alsbald in die Not, das Hervorgehen der Theorie einerseits 
aus der Lebenswelt ableiten, sie dann aber wiederum der Lebenswelt wieder gegenüberzustellen zu müssen. Es scheint daher ratsam, „Normal-,, und „Widerstimmigkeit" von Intentionen als lebensweltlich gleichursprünglich anzusetzen oder sie als Ex-post-Bestimmungen allenfalls in einem analytische Sinne zu verwenden. Ihr Zusammenwirken, nicht ihr Gegensatz ist Voraussetzung der Intentionalität. Keine der beiden lässt sich gegenüber der jeweils anderen prioritär oder fundierend auszeichnen. ${ }^{3}$

Auch wenn Blumenbergs husserlianische Verortung des Metaphorischen in der Lebenswelt nicht schlüssig zu der von ihm vorausgesetzten Anthropologie führt, die den Menschen mangelbedingt als eben ein symbolisierendes Tier oder Distanzierungswesen begreift (vgl. Blumenberg 1981 und Blumenberg 2006), so eröffnet sie doch die Möglichkeit zur Bestimmung ,absoluter Metaphern“, die sich dem „kristallisierten“ Sprachgebrauch entziehen und daher ihre metaphorische Qualität bewahren, auch wenn sie quasi-begrifflich verwendet werden. Diese Qualität besagt, um die weiter oben gegebene Beschreibung noch einmal aufzugreifen, dass sich solche Metaphern nicht in eine begrifflich erstarrte, formalisierbare oder terminologisch $\mathrm{zu}$ vereindeutigende, geschweige denn ontologisch oder metaphysisch konstruierbare Sprache eingemeinden lassen. Dadurch kann Philosophie als Umgangsweise mit solchen Metaphern selbst als eine Gabe und als Aufgabe bestimmt werden, die Metaphorizität solcher Metaphern aufzuweisen, zu dokumentieren oder, um es pathetisch auszudrücken, zu bezeugen. Umgekehrt lässt sich aus dieser Einsicht folgern, dass jeder Versuch, aus absoluten Metaphern Termini im oben genannten Sinne zu machen, zum Scheitern verurteilt sein wird, weil dieser Versuch Philosophie ,überfordern“ würde, um hier Blumenbergs Formulierung aufzugreifen. Ohne auf Blumenbergs anthropologische Festlegung des Menschen auf ein Mängelwesen und, in Reaktion darauf, ein diese Mängel durch Symbole kompensierendes animal zurückgreifen zu müssen, könnte man zumindest der absoluten Metapher jene zentrale und betörende, wenn auch unvermeidlicherweise immer wieder ,übersprungene“ und, wie Nietzsche

${ }^{3}$ Vgl. dazu bes. Müller 2005, 236ff. und zusammenfassend 239 „Letztlich hat Blumenberg keine einheitliche kulturanthropologische Theorie aufgestellt, sondern vielmehr versucht, seine metaphorologischen und phänomenologischen Untersuchungen anthropologisch zu fundieren." 
schreibt, „vergessene“ Kraft zuschreiben, die auf das Unsagbare geht, weil sie die terminologische Kristallisationskraft des Begriffs noch überschreitet und ein spezifisches „Weltverhältnis“ konstituiert. Man würde ihr auf diese Weise jene „verborgene Kraft“ zurückgeben, die Davidson aufgefallen ist, die er aber aufgrund der von ihm vorgenommenen Inklusion des Metaphorischen in eine auf ihre propositionalen Gehalte reduzierte Sprache nicht begreifen kann.

\section{III}

Im Unterschied zur analytischen Philosophie möchte Blumenberg die Geschichte der abendländischen Philosophie von Thales bis Wittgenstein vom Kommandohügel der Geschichte aus nachschreiben und dabei Nietzsches „bewegliches Herr von Metaphern“ immer wieder den jeweiligen Darstellungsbedürfnissen entsprechend neu in Stellung bringen (vgl. Müller 2005, 254ff.).

Wittgenstein spielt hierbei keineswegs zufällig eine bedeutende Rolle. Blumenberg hat ihm in seinem monumentalen Werk Höhlenausgänge, das natürlich mit Platon beginnt, das letzte Kapitel personenbezogene Kapitel gewidmet (Blumenberg 1989). Dass Wittgenstein als der vorerst letzte Höhlenbewohner erscheint, ist vielleicht historisch kontingent, aber gewiss nicht ohne Reiz - korrespondiert doch das berühmte Fliegenglas-Bild aus den Philosophischen Untersuchungen in markanter Weise mit Platons Höhlen-Gleichnis. Dem Wittgenstein'schen Bild vom Einschluss des Philosophen im gläsernen Gefängnis einer falsch gedeuteten Sprache hat Blumenberg 1989 einen eigenen Aufsatz gewidmet und darin nachzuweisen versucht, dass Wittgensteins philosophische Grunderfahrung in der „Angst vor letzten Ungewissheiten“ bestehe, wobei er das cartesianische Selbstvergewisserungsprogramm verworfen und sich deren „Vergeblichkeit“ als „Sorgen“ zu entledigen gesucht habe (Blumenberg 2001a, 213 und 244). Mit diesem an Husserl und Heidegger gemahnenden Motiv der Verwandlung lebensweltlicher Angst in philosophische Sorge bleibt Blumenberg seinem bereits erörterten fehlerhaften Stabilisierungsmodell der Beseitigung von „Widerstimmigkeit“ treu. Dasselbe gilt auch für Blumenbergs Studien zum Begriff der Unexaktheit bei Wittgenstein. Wie Bettine Menke vor kurzem gezeigt hat, fällt Blumenbergs Deutung, Wittgen- 
stein sei bestrebt, das Denken auf festem Grund und diesseits einer Grenze zum Sumpf der Unbestimmtheit zu halten, auch wenn dies zu willkürlichen Demarkationslinien führe, hinter dem zurück, was Wittgenstein in seinen Überlegungen verhandelt: Widerstimmigkeit und Normalstimmigkeit sind für Wittgenstein allenfalls zwei Pole in einem Sprach- und Handlungsfeld, innerhalb dessen sie ihre kontradiktorische Valenz entfalten - und nicht umgekehrt, wie Blumenberg unterstellt (vgl. dazu Menke 2009).

\section{IV}

Dass das metaphorisierende Verfahren Blumenbergs trotz seiner unübersehbaren erkenntnistheoretischen Problematik für die Erschließung der Philosophie Wittgensteins methodisch fruchtbar gemacht werden kann, möchte ich an einer Leitmetapher seines Denkens erläutern: der Metapher des Lichts. ${ }^{4}$ Ich möchte daraus den Vorschlag ableiten, auch die eindeutig nicht-metaphorisch intendierten Teile des Wittgenstein'schen Werks einer „metaphorisierenden“ Lektüreweise zu unterziehen, mit deren Hilfe die unentscheidbar scheinenden Debatten um dessen logische Konsistenz (in erster Linie des Frühwerks) selbst in ein neues Licht getaucht werden und dadurch wenn nicht gelöst, so doch sistiert werden können.

Das Motiv des Lichtentzugs, des Dunklen, Tiefen, der Gefangenschaft, der unüberwindlichen Grenze, der Hilflosigkeit etc. - und die entsprechenden Gegenbegriffe wie dazu - durchzieht bekanntlich das gesamte Werk Wittgensteins. Ilse Somavilla hat in ihrem Aufsatz „Wittgensteins Metapher des Lichts“ (Somavilla 2004) die entsprechenden Stellen aus Wittgensteins Gesamtwerk auf beeindruckende Weise zusammengestellt und damit einen Schatz aus überaus beeindruckenden Textstellen angelegt, der Blumenbergs Befunde in vielem ergänzt und in jeder Hinsicht bestätigen kann. Somavilla schreibt:

Nicht von ungefähr hat Wittgenstein sich immer wieder der Metapher des Lichts bedient - im Hinblick auf seine philosophischen Gedankengänge, doch auch im persönlichen Leben, wo er nach innerer moralischer Erneuerung, nach

\footnotetext{
${ }^{4}$ Blumenberg hat 1957 eine erste größere begriffsgeschichtliche Studie der Metapher des Lichts gewidmet, ohne freilich dabei auf Wittgenstein einzugehen (Blumenberg 2001b).
} 
einem Leben im Geist strebte, das für ihn identisch mit einem Leben im Licht war.

$[\ldots]$

174mal kommt der Begriff des Lichts in Wittgensteins Nachlass vor, doch in vielen Fällen nur in seinen aphorismenhaften Darstellungen (in den Philosophischen Untersuchungen) zur Veranschaulichung der Anwendung von Wörtern im alltäglichen Sprachgebrauch: in Sprachspielen, wie dem von ,licht” und „finster”, wo es um das Beibringen des Unterschieds dieser zwei Begriffe anhand von praktischen Beispielen geht (vgl. MS 113, 45v). Hier ist die philosophische Bedeutung irrelevant oder nur peripher; es überwiegt das Rhetorische. Wittgenstein verwendet bekannte Redewendungen wie „Licht auf etwas werfen”, „etwas ins richtige Licht rücken” und dergleichen: „Dies sollte ja darauf ein Licht werfen, was es heißt, etwas als Satz ansehen." (MS 112, Bd. VIII, 40r) Oder: „[...] das wirft ein Licht auf den Sinn der Ausdrücke ,Sinn haben' \& ,etwas mit dem Satz meinen'." (MS 112, 44r).

Die bildhaften Redewendungen dienen allenfalls zur lebhaften Untermalung seiner Beispiele, wenn auch seine Methode mit dem Ziel, philosophische Probleme zu klären, stets präsent ist und er das Bild des Lichts häufig mit einbezieht. Durch die adäquate Beschreibung mittels richtiger Wahl der Worte gelangen wir dazu, die Dinge zu verstehen, sie im richtigen Licht zu ,sehen”. (Somavilla 2004, 364)

Der von Somavilla vorgenommenen Trennung zwischen einem ,philosophisch irrelevanten“" und einem, im Gegensatz dazu, philosophisch bedeutsamen Gebrauch der Licht-Metapher bei Wittgenstein muss ich trotz aller Wertschätzung, die ich ihr und ihren sehr verdienstvollen Arbeiten und Text-Editionen etwa der Tagebücher Wittgensteins aus den 30er Jahren entgegenbringe, energisch widersprechen. Allerdings räumt sie selbst an anderer Stelle ihres Aufsatzes zur Lichtmetapher ein, dass für Wittgenstein Persönliches und Philosophisches ohnehin nicht klar $\mathrm{zu}$ trennen sind. Ich möchte diese Bemerkung ausdrücklich unterstreichen und im Folgenden an einem Beispiel radikalisieren. Sie zitiert Wittgensteins bewegenden Eintrag vom Frühjahr 1937 in sein Tagebuch: „Ich dachte gestern an den Ausdruck: , ein reines Herz'; warum habe ich keines? Das heißt doch: warum sind meine Gedanken so unrein!“ (MS 183, 216f.; Wittgenstein 1997a, 96) Um was für Gedanken handelt es sich? - Um persönliche? Sicherlich. Wir wissen, dass er sich Mitte der 30er Jahre genötigt fühlte, vor verschiedenen Personen ein Geständnis seiner Verfehlungen abzulegen - um alles ihm Mögliche getan zu haben, ein, reines Herz' zu bekommen, so wie es im 
Psalm 51,12 heißt, auf den sich Wittgenstein mit der Wendung, reines Herz' offensichtlich bezieht: „Schaffe in mir, Gott, ein reines Herz, und gib mir einen neuen, beständigen Geist. ${ }^{\text {“5 }}$

Doch gibt es zu viele Hinweise aus den Tagebüchern, dass Wittgenstein ,unreine“ philosophische Gedanken, wie es bereits Russell in einer ähnlichen Situation Jahrzehnte zuvor in Cambridge diagnostiziert hatte? Auf seine Frage erinnert sich Russell, der damalige Freund und spätere erbitterte Gegner Wittgensteins, ob er, Wittgenstein, beim Philosophieren über seine Sünden oder über die zu anstehenden sachlichen Probleme nachdenke, habe Wittgenstein geantwortet: Über beides (vgl. McGuinness 1988, 253). Für eine solche interne Verknüpfung von Persönlichem und Philosophischem spricht auch eine weitere Bemerkung Ilse Somavillas. Sie schreibt: „Neben den gängigen Redewendungen wird die Metapher des Lichts in Zusammenhang mit [der] philosophischen Tätigkeit verwendet als Bild für die Entwicklung seiner Gedanken. Der Unterschied zur philosophisch bedeutsamen Metapher ist dabei nur noch gering. ${ }^{\text {‘6 }}$

${ }^{5}$ Diese Bemerkungen stehen in folgendem Kontext:

11 Verbirg dein Antlitz vor meinen Sünden, und tilge alle meine Missetat.

12 Schaffe in mir, Gott, ein reines Herz, und gib mir einen neuen, beständigen Geist.

13 Verwirf mich nicht von deinem Angesicht, und nimm deinen Heiligen Geist nicht von mir.

14 Erfreue mich wieder mit deiner Hilfe, und mit einem willigen Geist rüste mich aus.

(zit. nach der Lutherbibel, rev. Standardausgabe 1984)

Die Veranlassung für Psalm 51, den vierten Bußpsalm, ist Davids heimtückischer Mordauftrag gegen Uria den Hethiter, seinen Feldherrn und zugleich Gatte Batsebas. David lässt Uria beseitigen, um Batseba zur Frau nehmen zu können. Erst als der Weise Nathan ihm ein Gleichnis vorträgt, erkennt David seine Schuld (vgl. Samuel II, 11-12). - Zu Wittensteins Lektüre des AT vgl. auch den Hinweis auf die (Buß)Psalmen-Lektüre in der Notiz vom 20.2.1937 in Denkbewegungen (Wittgenstein 1997a, 85).

${ }^{6}$ Somavilla stützt ihren Befund mit einem Beleg aus MS 107, 156: „Kann noch immer nicht ordentlich, oder gar nicht, arbeiten. Die philosophische Gegend meines Gehirns liegt noch immer im Dunkeln. Und erst wenn da wieder das Licht angezündet wird geht die Arbeit wieder an." 
Diese von Somavilla so genannte „Tätigkeit" ist es, die Wittgenstein im Sinn hat, wenn er in der Abhandlung schreibt, dass Philosophie keine Lehre, sondern eine Tätigkeit sei. Sie ist eine philosophische Übung, eine Askesis, nach deren erfolgreichem Vollzug man die Welt im richtigen Licht zu sehen vermag und nicht länger einer Sehhilfe, und sei es der $A b$ handlung selbst, bedürfe, wie es am Ende der Frühschrift bekanntlich heißt. Als ein weiteres Beispiel kann die Eintragung in MS 183 dienen:

Das Höchste aber, das ich zu erfüllen bereit bin, ist: ,fröhlich zu sein in meiner Arbeit'. [...] Was aber höher liegt, kann, oder will, ich nicht anstreben, ich kann es nur anerkennen [...]. // Es muss dazu, gleichsam, durch die Decke, den Plafond, unter dem ich arbeite, über den ich nicht steigen will, ein Licht durchschimmern. (MS 183, 168; Wittgenstein 1997a, 78)

Wittgenstein spielt hier wiederum auf das AT an, diesmal auf das Buch Kohelet. Es heißt dort in Kap. 3, 9-13:

9 Man mühe sich ab, wie man will, so hat man keinen Gewinn davon.

10 Ich sah die Arbeit, die Gott den Menschen gegeben hat, dass sie sich damit plagen.

11 Er hat alles schön gemacht zu seiner Zeit, auch hat er die Ewigkeit in ihr Herz gelegt; nur dass der Mensch nicht ergründen kann das Werk, das Gott tut, weder Anfang noch Ende.

12 Da merkte ich, dass es nichts Besseres dabei gibt als fröhlich sein und sich gütlich tun in seinem Leben.

13 Denn ein Mensch, der da isst und trinkt und hat guten Mut bei all seinem Mühen, das ist eine Gabe Gottes. ${ }^{7}$

Doch damit nicht genug des Bibelbezugs. An anderer Stelle desselben MS heißt es, dass er, um am Leben zu bleiben, ,ein neues Licht anderswoher" benötige, in welchem „keine Klugheit, oder Weisheit" sei (ebd., 232; Wittgenstein 1997a, 101). Dieses herbeigesehnte ,andere Leben“ aber sei „die Liebe, die menschliche Liebe, zum Vollkommenen. Und diese ist der Glaube“ (ebd., 233; Wittgenstein 1997a, 101f.). - Das ist sicherlich aus dem Hohelied der Liebe im ersten Korintherbrief des Paulus hergenommen. ${ }^{8}$ (Notabene: Wittgenstein trägt zu dieser Zeit in sein Tagebuch ein,

${ }^{7}$ Ich zitiere nach der revidierten Luther-Übersetzung von 1912.

${ }^{8}$ Vgl. 1 Kor 13:

8 Die Liebe höret nimmer auf, so doch die Weissagungen aufhören werden und die Sprachen aufhören werden und die Erkenntnis aufhören wird. 
dass er das „NT umschwirre wie die Motte das Licht“, und eine genauere Auswertung der Paulus-Briefe würde so manche weitere überraschende Einsicht in Wittgensteins Werk ermöglichen, auch wenn Wittgenstein durchaus kein Anhänger des Paulus war.) ${ }^{9}$

Ganz ähnlich formuliert es Wittgenstein in dem bereits zitierten MS 134:

Ist, was ich tue überhaupt der Mühe wert? Doch nur, wenn es von oben her ein Licht empfängt. Und ist es so, - warum sollte ich mich sorgen, daß mir die Früchte meiner Arbeit nicht gestohlen werden? Wenn, was ich schreibe, wirklich wertvoll ist, wie sollte man mir das Wertvolle stehlen? Ist das Licht von oben nicht da, so kann ich ja doch nur geschickt sein. (MS 134, 95; Wittgenstein 1997b, 114)

V

Ich habe mit diesen wenigen Beispielen die Metapher des Lichts bei Wittgenstein deshalb untersucht, weil sie gleichsam schlaglichtartig klar werden lässt, worin die Bedeutung von Metaphern für das Denken und das Werk Wittgensteins insgesamt bestehen könnte. Ein weiteres eklatantes Anwendungsfeld für eine Metaphorisierung in absolutem Sinne wäre gewiss auch das Bedeutungsfeld, das sich um die Sagen/Zeigen-Relation und den Terminus der Unsagbarkeit erstreckt. ${ }^{10}$

9 Denn unser Wissen ist Stückwerk, und unser Weissagen ist Stückwerk.

10 Wenn aber kommen wird das Vollkommene, so wird das Stückwerk aufhören. [...]

12 Wir sehen jetzt durch einen Spiegel in einem dunkeln Wort; dann aber von Angesicht zu Angesicht. Jetzt erkenne ich's stückweise; dann aber werde ich erkennen, gleichwie ich erkannt bin.

13 Nun aber bleibt Glaube, Hoffnung, Liebe, diese drei; aber die Liebe ist die größte unter ihnen.

${ }^{9}$ Dies betrifft insbesondere die Lehre von der Gnadenwahl, die Paulus in Römer 9 knapp darlegt.

${ }^{10}$ Blumenberg hat bei seiner Ausarbeitung des Begriffs der ,absoluten Metapher“ in seinen Paradigmen (1960) gewiss nicht an Wittgenstein gedacht; er bezog sich vor allem auf Cassirer, Heidegger und Husserl. Vgl. Heidenreich (2005), bes. S. 95105. 
Ich möchte aus meinen Befunden einen für manchen nur schwer nachvollziehbaren Vorschlag zu einer nochmaligen kritischen Lektüre des Wittgenstein'schen Werkes, namentlich der Abhandlung, unterbreiten, nämlich mit den Augen einer um das wenig tragfähige normativanthropologische Fundament bereinigten, auf den Blumenberg'schen Metaphernbegriff konzentrierten Lektüre vorzunehmen.

Der Beitrag Blumenbergs zu Wittgenstein ist meines Erachtens bisher noch zu wenig in die Wittgensteinforschung eingegangen. Dies ist umso bedauerlicher, als Blumenberg mit seinen Überlegungen zur ,absoluten“ Metapher helfen kann, die ,dunklen' Passagen des Wittgenstein'schen Frühwerks philosophisch aufzuklären (und damit z.B. auch den epischbreiten Debatten um den Begriff des „Unsinns“ im Tractatus einen neuen, vielleicht sogar befreienden Akzent zu geben). Zugleich kann man an Blumenbergs Überlegungen zur Unbegrifflichkeit zeigen, dass eine anthropologische Fundierung der Metapherntheorie auf fast unlösbare Probleme stößt. Blumenbergs anthropologisierende Einstellung gleicht eher der eines Insektenkundlers, wenn er in seinen historischen Gesamtschauen Wittgenstein gleichsam entomologisch klassifiziert, um ihn sodann wie einen Käfer in das dunkle Innere der philosophiegeschichtlichen Schachtel zurückzulegen.

Doch kann man Blumenbergs Einsichten auch anders verwenden. Sie geben uns einen Hinweis darauf, dass in Wittgensteins Texten ein unüberschaubar großes Heer von Metaphern in Marsch gesetzt wird. Fast das gesamte in philosophischen Dienst genommene Argumentationspersonal besteht aus Metaphern - angefangen mit der dem begrifflichen Arsenal, das er für die Isomorphiethese zwischen Welt und Bild der Frühschrift aufbietet und endend mit der Weigerung in den späten Schriften, überhaupt noch von festen begrifflichen Grenzen, Definitionen zu sprechen oder in der Sprache der Formalisierungen, Universalisierungen und logischen Ableitungen zu sprechen. Eine daraus abzuleitende These, für die ich auf Kritik und Widerspruch gefasst bin, würde lauten: Wittgensteins Aufklärungsund Korrekturarbeit an den Fehlern und Irrtümern der Philosophie besteht darin, anscheinend geronnene Begriffe wieder beweglich zu machen, sie als Metaphern zu lesen und damit den Nimbus ihrer Kristallreinheit, mit dem Ausdrucks Blumenbergs, den ich eingangs aus der Einleitung des His- 
torischen Wörterbuchs der Philosophie zitierte, ihre „Kristallisation“ und damit sprachliche Unbeweglichkeit zu nehmen.

Unter einem solchen Blickwinkel würde zugleich deutlich, welche Exklusionen die Inklusion Philosophie in die Sprache miterzeugt. Sie entzieht Ästhetik, Ethik und Religion dem Bereich der Sagbarkeit; sie überführt Philosophie als terminologisch gegen Metaphern immunisierte Tätigkeit der Unsinnigkeit, rehabilitiert sie freilich als Tätigkeit der Metaphorisierung zum Zwecke einer existenziellen Klärung, die sich nicht anders als in einer metaphorischen Spur manifestieren kann. Wie Nietzsche und Blumenberg gezeigt haben, liegt in diesem Entzug immer eine doppelte Bewegung: Durch sie und in ihr werden - um hier einen Lieblingsausdruck Wittgensteins zu verwenden - ,kristallisierte“ Überzeugungen wieder verflüssigt - diese Bewegung entzieht Gewissheit und Evidenz, sie unterminiert das Projekt der Petrifizierung von philosophischen Termini, weil und indem sie das als Metapher erweist, was als Begriff daherkommt.

Zugleich ist der Entzug, den uns Wittgenstein mit seiner Grenzziehung sinnvollen Sprechens zumutet, zugleich doch auch eine Gabe. Indem er philosophische Sätze auf den rauen Boden ihrer metaphorischen Herkunft zurückführt und damit das bisherige Philosophieren als Entziehung dieser metaphorischen Herkunft deutlich werden lässt, überreicht Wittgenstein zugleich eine Gabe: etwa die, die Welt richtig zu sehen, Friede in Gedanken zu erzielen, sich im alten Chaos wohl zu fühlen, das Philosophieren abbrechen zu können, wann man will, nicht länger der Tröpfcheninfektion der Metaphysik zu erliegen und so fort - besitzt damit nicht das Konzept der ,absoluten Metapher“ nicht vielleicht eine noch verborgene, aber nichtsdestoweniger nachhaltige Kraft für das philosophische Denken? 


\section{Literatur}

Arnswald, Ulrich / Kertscher, Jens / Kroß, Matthias (Hg.) 2004: Wittgenstein und die Metapher. Berlin: Parerga.

Blumenberg, Hans 1960/1999: Paradigmen zu einer Metaphorologie. Frankfurt a. M.: Suhrkamp.

Blumenberg, Hans 1979: Schiffbruch mit Zuschauer. Paradigma einer Daseinsmetapher. Frankfurt a. M.: Suhrkamp.

Blumenberg, Hans 1981: „Anthropologische Annäherung an die Aktualität der Rhetorik“. In: Wirklichkeiten in denen wir leben. Stuttgart: Reclam, 104-136.

Blumenberg, Hans 1989: Höhlenausgänge. Frankfurt a. M.: Suhrkamp.

Blumenberg, Hans 2001a: „Im Fliegenglas“ [1989]. In: Ästhetische und metaphorologische Schriften. Auswahl und Nachwort von Anselm Haverkamp. Frankfurt a. M.: Suhrkamp, 213-244.

Blumenberg, Hans 2001b: „Licht als Metapher der Wahrheit. Im Vorfeld der philosophischen Begriffsbildung“" [1957]. In: Ästhetische und metaphorologische Schriften. Auswahl und Nachwort von Anselm Haverkamp. Frankfurt a.M.: Suhrkamp, 139-171.

Blumenberg, Hans 2006: Beschreibung des Menschen. Hrsg. von Sommer, Manfred. Frankfurt a. M.: Suhrkamp.

Davidson, Donald 1998: „Was Metaphern bedeuten“ [1976]. In: Haverkamp, Anselm (Hg.): Die paradoxe Metapher. Frankfurt a. M.: Suhrkamp, 49-75.

Glock, Hans-Johann 2008: What is Analytic Philosophy? Cambridge: Cambridge UP.

Grassi, Ernesto 1992: Die unerhörte Metapher. Frankfurt a. M.: Hain.

Haverkamp, Anselm (Hg.) 1998: Die paradoxe Metapher. Frankfurt a. M.: Suhrkamp.

Haverkamp, Anselm 2007: Metapher. Die Ästhetik in der Rhetorik. München: Fink.

Heidenreich, Felix 2005: Mensch und Moderne bei Hans Blumenberg. München: Fink.

Kertscher, Jens 2004: „Von der Metapher zur Bildmetapher. Überlegungen im Anschluss an Davidson und Wittgenstein“. In: Arnswald et al. 2004, 165-194.

Konersmann, Ralf (Hg.) 2007: Wörterbuch der philosophischen Metaphern. Darmstadt: Wissenschaftliche Buchgesellschaft.

McGuinness, Brian 1988: Wittgenteins frühe Jahre. Frankfurt a. M.: Suhrkamp.

Menke, Bettine 2009: „Sumpf und Mauer. Versuche zu einer Philosophie der Unbestimmtheit". In: Haverkamp Anselm / Mende, Dirk (Hg.): Metaphorologie. Zur Praxis und Theorie. Frankfurt a. M.: Suhrkamp, 316-338. 
Müller, Oliver 2005: Sorge um die Vernunft. Hans Blumenbergs phänomenologische Anthropologie. Paderborn: mentis.

Nietzsche, Friedrich 1978: „Jenseits von Gut und Böse“. In: Kritische Studienausgabe, Bd. 5. Berlin / München: DeGruyter / dtv, 9-243.

Nietzsche, Friedrich 1984: „Über Wahrheit und Lüge im außermoralischen Sinn“. In: Kritische Studienausgabe, Bd. 1, Berlin / München: DeGruyter / dtv.

Ricœur, Paul 1986: Die lebendige Metapher. München: Fink.

Ritter, Joachim et al. (Hg.) 1971: Historisches Wörterbuch der Philosophie, Bd. 1. Basel: Schwabe \& Co.

Somavilla, Ilse 2004: „Wittgensteins Metapher des Lichts“, in: Arnswald et al., 361385 .

Steiner, George 1990: Von realer Gegenwart. Hat unser Sprechen Inhalt? München: Hanser.

Taureck, Bernhard W. F. 2004: Metaphern und Gleichnisse in der Philosophie. Frankfurt a. M.: Suhrkamp.

Wittgenstein, Ludwig 1997a: Denkbewegungen. Tagebücher 1930-1932/1936-1937. Bd. 1, hrsg. von Ilse Somavilla. Innsbruck: Haymon.

Wittgenstein, Ludwig 1997b: Vermischte Bemerkungen. Eine Auswahl aus dem Nachlass. Hrsg. von Georg Henrik von Wright unter Mitarbeit von Heikki Nyman. Neubearbeitung des Textes durch Alois Pichler. Frankfurt a. M.: Suhrkamp. 